\title{
The Effect of Smoking on COVID-19 Symptom Severity: Systematic Review and Meta-Analysis
}

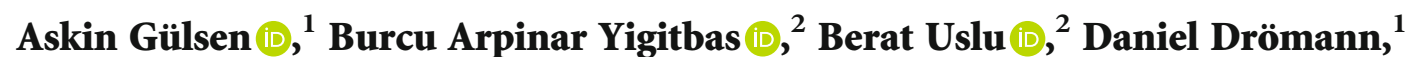 \\ and Oguz Kilinc $\mathbb{1}^{3}$ \\ ${ }^{1}$ Department of Pneumology, Airway Research Center North (ARCN), Member of the German Center for Lung Research (DZL), \\ University of Luebeck, Germany \\ ${ }^{2}$ Department of Pneumology, Yedikule Chest Disease and Thoracic Surgery Hospital, Istanbul, Turkey \\ ${ }^{3}$ Department of Pneumology, Dokuz Eylül University, Izmir, Turkey
}

Correspondence should be addressed to Askin Gülsen; askingulsen@hotmail.com

Received 2 June 2020; Accepted 19 August 2020; Published 9 September 2020

Academic Editor: Roberto Walter Dal Negro

Copyright () 2020 Askin Gülsen et al. This is an open access article distributed under the Creative Commons Attribution License, which permits unrestricted use, distribution, and reproduction in any medium, provided the original work is properly cited.

Background. Coronavirus disease 2019 (COVID-19) is caused by severe acute respiratory syndrome coronavirus 2 (SAR2-COV-2) and was first identified in Wuhan, China, in December of 2019, but quickly spread to the rest of the world, causing a pandemic. While some studies have found no link between smoking status and severe COVID-19, others demonstrated a significant one. The present study aimed to determine the relationship between smoking and clinical COVID-19 severity via a systematic meta-analysis approach. Methods. We searched the Google Scholar, PubMed, Scopus, Web of Science, and Embase databases to identify clinical studies suitable for inclusion in this meta-analysis. Studies reporting smoking status and comparing nonsevere and severe patients were included. Nonsevere cases were described as mild, common type, nonintensive care unit (ICU) treatment, survivors, and severe cases as critical, need for ICU, refractory, and nonsurvivors. Results. A total of 16 articles detailing 11322 COVID-19 patients were included. Our meta-analysis revealed a relationship between a history of smoking and severe COVID-19 cases (OR $=2.17 ; 95 \%$ CI: $1.37-3.46 ; P<.001)$. Additionally, we found an association between the current smoking status and severe COVID-19 (OR = 1.51; 95\% CI: 1.12-2.05; $P<.008)$. In 10.7\% (978/9067) of nonsmokers, COVID-19 was severe, while in active smokers, severe COVID-19 occurred in 21.2\% (65/305) of cases. Conclusion. Active smoking and a history of smoking are clearly associated with severe COVID-19. The SARS-COV-2 epidemic should serve as an impetus for patients and those at risk to maintain good health practices and discontinue smoking. The trial is registered with the International Prospective Register of Systematic Reviews (PROSPERO) CRD42020180173.

\section{Introduction}

Coronavirus disease 2019 (COVID-19), which is caused by severe acute respiratory syndrome coronavirus 2 (SAR2COV-2), was first identified in Wuhan, China, in December of 2019. It has subsequently spread across the world, causing a global pandemic. This highly contagious disease has thus far infected 23.4 million people worldwide and killed approximately 808000 patients, yielding a case fatality rate (CFR) that varies between 0.7 and $12.7 \%$ (average: $3.4 \%$ ) $[1,2]$.
COVID-19 primarily targets lung epithelial cells, causing viral pneumonia and acute respiratory distress syndrome (ARDS), especially in elderly patients. Therefore, mortality is higher in the elderly and in patients with at least one accompanying comorbid disease [3]. In the last report issued by the Centers for Disease Control and Prevention Institute, the incidence of respiratory disease was $9.2 \%$ in patients diagnosed with a severe COVID-19 clinical course [4]. Chronic obstructive pulmonary disease (COPD) and asthma are also common comorbidities in severe cases and are reported in 
$10.8 \%$ and $17.0 \%$, respectively, of hospitalized patients aged $\geq 18$ years with COVİD-19 [5].

However, it has been reported that COVID-19 progresses more severely in COPD patients [6]. Given that smoking plays an important role in the etiopathogenesis of COPD, it may have a similar effect on symptoms. In a recent metaanalysis of smoking and COVID-19 severity, smoking was found to not increase the severity of COVID-19 (odds ratio [OR], 1.69; 95\% Cl: 0.41-6.92). However, only five studies were included in this meta-analysis, and heterogeneity among the studies was low $\left(I^{2}=38 \%\right)$ [7].

In another meta-analysis involving more studies, COVID-19 severity was found to increase with smoking (OR, 1.97; 95\% Cl: 0.95-4.10) [6]. To increase the statistical power of this analysis, case reports and series were also included, not just case-control studies. As previously, the heterogeneity of the studies included here was also low $\left(I^{2}=44 \%\right)$ [6]. The different results of both meta-analyses create confusion about the issue of smoking status.

Given diverging findings in the existing literature, we systematically reviewed English-language studies to investigate whether smoking was associated with a more severe clinical course of COVID-19.

\section{Material and Methods}

All guidelines listed in the Preferred Reporting Items for Systematic Reviews and Meta Analyses (PRISMA) statement were followed in conducting this meta-analysis [8]. Our methodological systematic review and meta-analysis of pooled case-control studies were recorded in the International Prospective Register of Systematic Reviews (https://www.crd .york.ac.uk/prospero; registration number: CRD42020180173).

2.1. Study Search Strategy. We searched the Google Scholar, PubMed, Scopus, Web of Science, and Embase databases to identify clinical studies suitable for inclusion here. Only English-language articles published between December 2019 and April 15, 2020, were included. Case series/reports, commentaries, reviews, and editorial letters were excluded. Unpublished articles were not searched, but "ahead of print" and "first only" articles with a DOI link in the given database were included. The following search terms were used: "COVID-19," OR "SARS-COV-2," OR "novel coronavirus (nCOV)," AND “smoking," OR “tobacco," AND “clinical features," OR “characteristic," AND "severity," OR "severe".

2.2. Inclusion and Exclusion Criteria. Inclusion criteria were as follows: (i) studies that examined COVID-19 patients older than 18 years and diagnosed COVID-19 according to WHO criteria; (ii) observational, cross-sectional, prospective, or retrospective studies; (iii) studies which compared smokers with mild and severe COVID-19; (iv) studies comparing smokers with survivors and nonsurvivors of COVID-19; and (v) studies comparing smokers who developed COVID-19 that required intensive-care unit (ICU) and non-ICU treatment.

Exclusion criteria were as follows: (i) studies from the same medical center that did not contribute to meta-analysis, (ii) non-English-language studies, (iii) case reports/series, (iv) reviews, (v) editorial letters, (vi) studies lacking a fulltext (unavailable or not yet published), (vii) studies without a DOI, and (viii) studies with small sample sizes $(<50$ patients) because of low statistical power.

2.3. Data Extraction. A researcher (AG) scanned study titles and abstracts obtained via an initial database search and included relevant ones in a secondary pool. Next, two independent researchers (AG and BU) evaluated the full text of these articles to determine whether they met study inclusion criteria. Any disputes were resolved by discussion and negotiation with a third investigator (BAY). Agreed-upon studies were included in the final meta-analysis.

The following data were obtained from all studies: title, first author, publication year, location, sample size, age (median), history of smoking (former or current), and distribution of nonsevere or severe COVID-19 status. Given these groups, we were able to compare nonsevere (mild, common type, non-ICU, survivors) and severe (critical, severe, ICU, nonsurvivors) COVID-19 cases.

2.4. Risk of Bias (Quality) Assessment. Two researchers (BAY and BU) evaluated the collected articles using the NewcastleOttawa scale (NOS) for study quality and risk of bias [9]. This scale evaluates three aspects of each study: (i) selection (0 to 4 points), (ii) comparability (0 to 2 points), and (iii) detection of the outcome of interest ( 0 to 3 points). According to the obtained total score, study quality was rated as follows: $0-3$ points (poor), 4-6 points (intermediate), and 7-9 points (high). Studies with a high risk of bias were excluded during the meta-analysis phase.

2.5. Statistical Analyses. OpenMeta Analyst version 10.10 (https://www.cebm.brown.edu/open_meta) was used to calculate odds ratios with $95 \%$ confidence intervals, which are depicted using forest plots. Quantitative numbers were measured in terms of total numbers and percentages $(n, \%)$. The odds ratio of smokers among nonsevere COVID-19 and severe COVID-19 cases, as well as survivors and nonsurvivors, was calculated. Heterogeneity was evaluated with Cochran's Q, $\chi^{2}$, and the Higgins $I^{2}$ test. The Higgins $I^{2}$ test uses a fixed effect model when $I^{2}<50 \%$, and a random effects model when $I^{2}>50 \%$. When heterogeneity was detected, a sensitivity adjustment was made to determine its source. This procedure was performed by leaving one study out of the analysis at a time, with a fixed effect model used after excluding heterogeneity. Publication bias was evaluated with Statsdirect version 3.2.10 (StatsDirect Ltd, Cambridge, UK) and visualized using Begg's funnel plots and Egger's test. $P$ values (two-sided) were considered statistically significant if $P<.05$.

\section{Results}

Our initial search of international databases using the keywords described above yielded 265 articles. After duplicate articles were excluded, 197 articles remained. After article titles and abstracts were evaluated for appropriateness, a final 34 articles were found to meet study inclusion criteria. In 


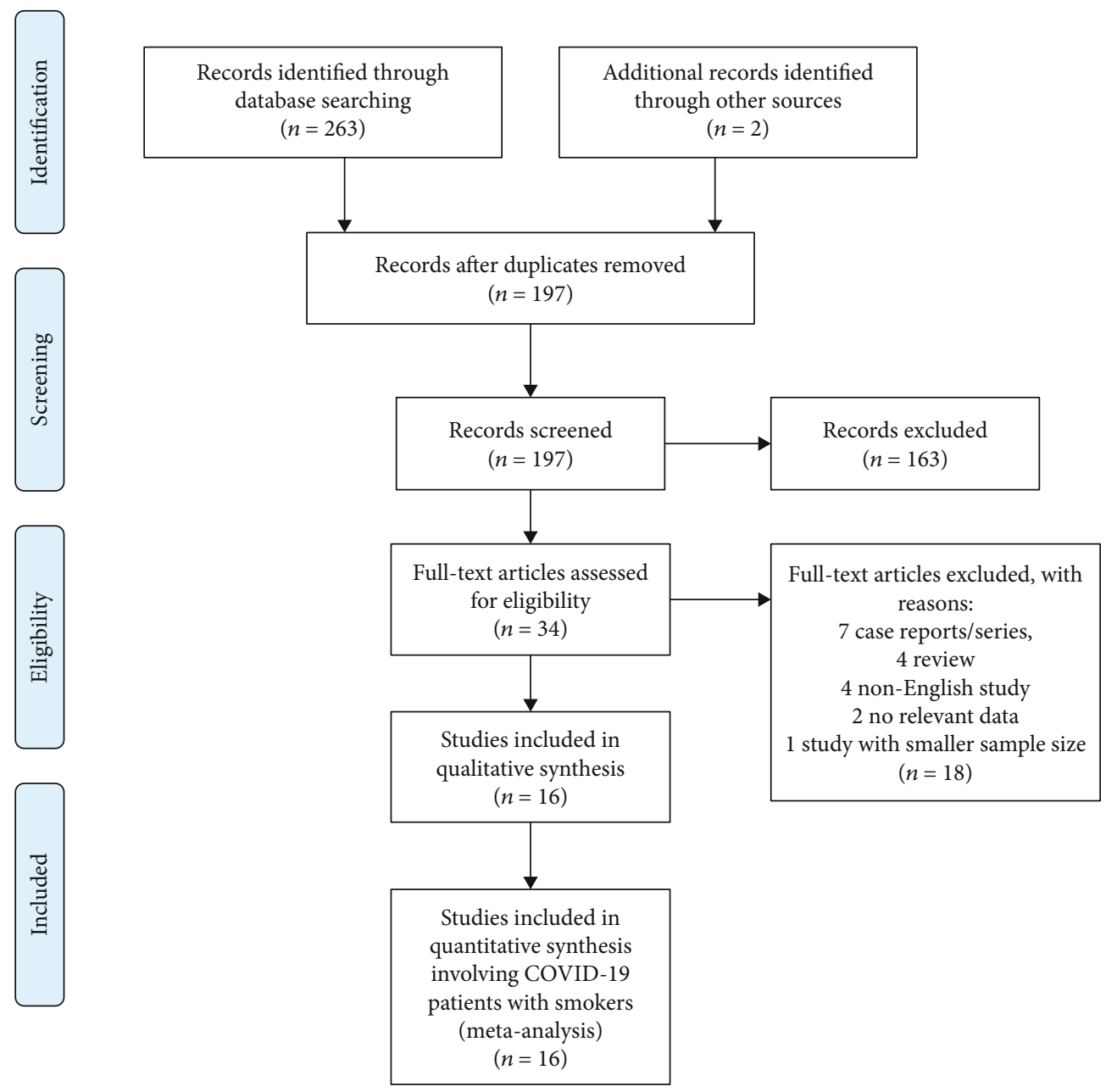

Figure 1: PRISMA flow diagram of study selection procedures.

addition, 18 of the studies for which full texts were obtained were excluded because they met exclusion criteria. A total of 16 articles met all our criteria. The relevant PRISMA study flow chart is shown in Figure 1. A quality analysis of the pooled articles included in this meta-analysis was done by applying NOS. As a result, overall quality was found to be moderate (NOS score min: 5, max: 8). Results of a quality analysis of the pooled articles are available in Table 1.

3.1. Features of the Included Studies. In the initial metaanalysis pool, 11322 patients with COVID-19 from 16 studies $[4,10-24]$ were included. However, given CDC reporting guidelines [4], 525 patients whose hospitalization statuses were reported as "unknown" were excluded from the final analysis, yielding a final 10797 patients. Of these, 9414 (87.2\%) were nonsevere COVID-19 patients, while 1383 (12.8\%) were severe or critical COVID-19 patients who required intensive care. Among the severe or critical patients, smoking prevalence ranged from 3.6 to 19.9\% (average: $8.4 \%)$. While nine studies provided current smoker information, three provided both current smoker and former smoker information, and seven provided only historical smoking information. All of the included studies were retrospective (six multicenter and 10 single-center). A summary of the studies included in this meta-analysis is available in Table 2.

3.2. History of Smoking and COVID-19 Severity. The prevalence of smoking among patients with mild to moderate COVID-19 was 493/9414 (5.2\%), while among severe and critical cases, it was $173 / 1383(12.5 \%)$. A meta-analysis revealed a significant relationship between smoking history and severe COVID-19 (random effect model, OR = 2.17; 95\% CI:1.37-3.46; $P<.001$ ) (Table 3) (Figure 2(a)). A funnel plot was used to evaluate publication bias and revealed substantial heterogeneity between the pooled studies $\left(I^{2}=\right.$ $71.4 \%$; $P<.001$ ) (Figure $2(\mathrm{~b})$ ). A sensitivity analysis conducted after removing two studies (Qi et al. [16] and Zhang et al. [23]), which were primary causes of heterogeneity, revealed that smoking was significantly related to COVID19 severity (fixed effect model, OR $=1.85$; 95\% CI:1.502.29; $P<.001$ ) (Figure 2(c)). Furthermore, this updated analysis had low heterogeneity $\left(I^{2}=40.6 \%\right.$; $\left.P=.06\right)$, while a funnel plot and Egger's test revealed no publication bias $\left(\operatorname{tau}^{2}=-0.87, P=.16\right)$ (Figure 2(d)).

3.3. Smoking Status and COVID-19 Severity. Only 10 of the pooled studies provide information about current 
TABLe 1: Newcastle-Ottawa scale for quality assessment and risk of bias.

\begin{tabular}{|c|c|c|c|c|c|}
\hline Study & Year & Case selection (max. 4) & Comparability (max. 2) & Exposure/outcome (max. 3) & Total score \\
\hline CDC report $[4]$ & 2020 & $* * * *$ & $*$ & $* *$ & 7 \\
\hline Chen Q [10] & 2020 & $* * *$ & $*$ & $* *$ & 6 \\
\hline Feng Y [11] & 2020 & $* *$ & $*$ & $* * *$ & 6 \\
\hline Goyal P [12] & 2020 & $* *$ & - & $* * *$ & 5 \\
\hline Guan WJ [13] & 2020 & $* *$ & $* *$ & $* *$ & 6 \\
\hline Liu W [14] & 2020 & $* *$ & $*$ & $*$ & 7 \\
\hline Mo P [15] & 2020 & $* *$ & $*$ & $* * *$ & 6 \\
\hline Qi D [16] & 2020 & $* * *$ & $*$ & $* * *$ & 7 \\
\hline Shi Y [17] & 2020 & $* *$ & $*$ & $* *$ & 5 \\
\hline Wang R [18] & 2020 & $* * *$ & $*$ & $* *$ & 6 \\
\hline Wan S [19] & 2020 & $* * *$ & $*$ & $* * *$ & 7 \\
\hline Yang X [20] & 2020 & $* * *$ & $* *$ & $* * *$ & 8 \\
\hline Yu X [21] & 2020 & $* * *$ & $* *$ & $* *$ & 7 \\
\hline Zhang JJ [22] & 2020 & $* * *$ & $* *$ & $* *$ & 7 \\
\hline Zhang R [23] & 2020 & $* * *$ & $*$ & $* *$ & 6 \\
\hline Zhou F [24] & 2020 & $* * *$ & $*$ & $* * *$ & 7 \\
\hline
\end{tabular}

TABLE 2: Features of the studies included in the meta-analysis.

\begin{tabular}{|c|c|c|c|c|c|c|c|c|}
\hline First author & Location of patients & Study design & Comparison & $n$ & Age, year & $\begin{array}{l}\text { Prevalence } \\
\text { of smoking }\end{array}$ & $\begin{array}{c}\text { Former } \\
\text { smokers }\end{array}$ & $\begin{array}{l}\text { Current } \\
\text { smokers }\end{array}$ \\
\hline CDC report $[4]$ & - & $\mathrm{R}, \mathrm{MC}$ & Non-ICU vs. ICU & 7162 & - & $261(3.6)$ & $165(2.3)$ & $96(1.3)$ \\
\hline Chen Q [10] & Taizhou, China & $\mathrm{R}, \mathrm{O}, \mathrm{SC}$ & Nonsevere vs. severe & 145 & 47.5 & $15(10.3)$ & - & - \\
\hline Feng Y [11] & $\begin{array}{l}\text { Wuhan, Anhui, } \\
\text { Shanghai }\end{array}$ & $\mathrm{R}, \mathrm{O}, \mathrm{MC}$ & Moderate vs. severe, critical & 454 & 53.0 & $44(9.7)$ & - & - \\
\hline Goyal P [12] & Newyork, USA & $\mathrm{R}, \mathrm{MC}$ & Non-IMV vs. IMV & 393 & 62.2 & $20(5.1)$ & - & $20(5.1)$ \\
\hline Guan WJ [13] & 552 hospitals in China & $\mathrm{R}, \mathrm{MC}$ & Nonsevere vs. severe & 1085 & 47.0 & $158(12.6)$ & $21(1.9)$ & $137(12.6)$ \\
\hline Liu W [14] & Wuhan, China & $\mathrm{R}, \mathrm{SC}$ & Stabilization vs. progression & 78 & 38.0 & $5(6.4)$ & - & - \\
\hline Mo P [15] & Wuhan, China & $\mathrm{R}, \mathrm{SC}$ & General vs. refractory & 155 & 54.0 & $6(3.9)$ & - & $6(3.9)$ \\
\hline Qi D [16] & Chongqing, China & $\mathrm{R}, \mathrm{MC}$ & Nonsevere vs. severe & 267 & 48.0 & $53(19.9)$ & - & - \\
\hline Shi Y [ 17] & Zhejiang, China & $\mathrm{R}$ & Mild vs. severe & 487 & 46.0 & $40(8.2)$ & - & - \\
\hline Wang R [18] & Fuyang, China & $\mathrm{R}, \mathrm{SC}$ & Noncritical vs. ritical & 125 & 49.4 & $16(12.8)$ & - & $16(12.8)$ \\
\hline Wan S [19] & Chongqing, China & $\mathrm{R}, \mathrm{SC}$ & Mild vs. severe & 135 & 47.0 & $9(6.7)$ & - & $9(6.7)$ \\
\hline Yang X [20] & Wuhan, China & $\mathrm{R}, \mathrm{O}, \mathrm{SC}$ & Survivors vs. nonsurvivors & 52 & 51.9 & $2(10.0)$ & - & - \\
\hline Yu X [21] & Shanghai, China & $\mathrm{R}, \mathrm{SC}$ & Nonsevere vs. severe & 333 & 50.0 & $26(7.8)$ & - & - \\
\hline Zhang JJ [22] & Wuhan, China & $\mathrm{R}, \mathrm{SC}$ & Nonsevere vs. severe & 140 & 57.0 & $9(6.4)$ & $7(5.0)$ & $2(1.4)$ \\
\hline Zhang R [23] & Wuhan, China & $\mathrm{R}, \mathrm{SC}$ & Common type vs. severe & 120 & 45.4 & $6(5.0)$ & - & $6(5.0)$ \\
\hline Zhou F [24] & Wuhan, China & $\mathrm{R}, \mathrm{O}, \mathrm{MC}$ & Nonsurvivor vs. survivor & 191 & 56.0 & $11(6.0)$ & - & $11(6.0)$ \\
\hline
\end{tabular}

Data are presented as “ $n$ ” or " $n(\%)$." Age-related data are given as "median year." $n$ : population; R: retrospective; CS: cross-sectional; O: observational; IMV: invasive mechanic ventilation; ICU: intensive care unit; SC: single-center; MC: multicenter.

smoking, specifically $(n=305)$. Current smoking prevalence in patients with mild to moderate COVID-19 was 229/7850 $(2.9 \%)$, while in severe or critical cases, it was $66 / 1134$ $(5.8 \%)$. However, in $10.7 \%(978 / 9067)$ of nonsmokers, COVID-19 disease was severe, while in active smokers, it was severe in $21.2 \%(65 / 305)$ of cases (Table 4$)$. This clearly demonstrates a significant relationship between current smoking and severe COVID-19 patients (fixed effect model, $\mathrm{OR}=1.51 ; 95 \% \mathrm{CI}: 1.12-2.05 ; z=2.65 ; P=.008)$, with an approximate 1.5-fold increased risk of ICU admission, symptom, severity, and mortality in smokers (Figure 3(a)). In this analysis, we used a fixed effect model due to the low level 
TABLE 3: The relationship between history of smoking and the severity of COVID-19.

\begin{tabular}{|c|c|c|c|c|c|c|}
\hline \multirow{2}{*}{$\begin{array}{l}\text { Studies } \\
\text { Authors }\end{array}$} & \multicolumn{3}{|c|}{ Severe COVID-19 with history of smoking } & \multicolumn{3}{|c|}{ Nonsevere COVID-19 with history of smoking } \\
\hline & Event & Total & $\%$ & Event & Total & $\%$ \\
\hline CDC report $[4]$ & 38 & 457 & 8.3 & 208 & 6180 & 3.3 \\
\hline Chen et al. [10] & 3 & 43 & 7.0 & 12 & 102 & 11.0 \\
\hline Feng et al. [11] & 17 & 121 & 14.0 & 27 & 333 & 8.1 \\
\hline Goyal et al. [12] & 6 & 130 & 4.6 & 14 & 263 & 5.3 \\
\hline Guan et al. [13] & 38 & 172 & 22.0 & 120 & 913 & 13.0 \\
\hline Liu et al. [14] & 3 & 11 & 27.3 & 2 & 67 & 2.0 \\
\hline Mo et al. [15] & 4 & 85 & 4.7 & 2 & 70 & 2.9 \\
\hline Qi et al. [16] & 31 & 50 & 62.0 & 22 & 217 & 10.1 \\
\hline Shi et al. [17] & 6 & 49 & 12.2 & 34 & 438 & 7.8 \\
\hline Wang et al. [18] & 7 & 25 & 28.0 & 9 & 100 & 9.0 \\
\hline Wan et al. [19] & 1 & 40 & 2.5 & 8 & 95 & 8.4 \\
\hline Yang et al. [20] & 0 & 32 & 0 & 2 & 20 & 10.0 \\
\hline Yu et al. [21] & 2 & 26 & 8.0 & 24 & 307 & 8.9 \\
\hline Zhang et al. [22] & 6 & 58 & 10.3 & 3 & 82 & 3.6 \\
\hline Zhang et al. [23] & 6 & 30 & 20.0 & 0 & 90 & 0 \\
\hline Zhou et al. [24] & 5 & 54 & 9.0 & 6 & 137 & 4.0 \\
\hline Total & 173 & 1383 & 12.5 & 493 & 9414 & 5.2 \\
\hline
\end{tabular}

of heterogeneity $\left(I^{2}=48.9 \% ; P=.40\right)$. A funnel pilot and Egger's test revealed no publication bias $\left(\operatorname{tau}^{2}=0.22, P=\right.$ .77) (Figure 3(b)).

A sensitivity analysis conducted after removing one study (Zhang et al. [23]) revealed a similar relationship with severe COVID-19 (fixed effect model; OR $=1.36$; 95\% CI: $1.00-$ $1.87 ; P=0.05)$ and low heterogeneity $\left(I^{2}=34.0 \% ; P=.14\right)$ (Figure 3(c)). A funnel plot and Egger's test further revealed no publication bias $\left(\operatorname{tau}^{2}=-0.31, P=.67\right.$ ) (Figure 3(d)).

\section{Discussion}

The present meta-analysis contained 16 studies and revealed that those with a history of smoking and active smokers had significantly increased risk for severe COVID-19. Previous studies have reported a similar result $[14,18,23]$. In addition, one prior meta-analysis reported that disease severity increased given a history of smoking (OR: 1.98) [6]. Our analysis adds to this growing consensus in the literature, invoking more studies and examining both a history of smoking and current smoking status in risk for increased COVID-19 severity.

Five prior studies have reported results which disagree with those presented here-namely, no significant difference between patients with and without a smoking history in terms of COVID-19 severity $[10,15,21,22,24]$. In addition, a meta-analysis performed by Lippi et al. [7] failed to find a relationship between active smoking and severe COVID-19 on Chinese patients, and another meta-analysis indicates that active smoking is not a predisposing factor for hospitalization [25]. First of all, both meta-analysis [7, 25] was not systematic and included no assessment of publication bias or study quality. As such, it should be considered only preliminary. Hence, the present systematic meta-analysis offers a more detailed view as it covers 16 studies. The heterogeneity was low to moderate, and after sensitivity adjustments, the prevalence of a history of smoking was found to be $5.2 \%$ in nonsevere COVID-19 cases and $12.5 \%$ in severe cases. Furthermore, the prevalence of active smoking was $2.9 \%$ in nonsevere COVID-19 cases and 5.8\% in severe cases. Therefore, severe COVID-19 was observed almost 1.5 to 2 times more frequently in history of smoking and current smoking groups. There are studies $[4,11,14,16,18,23]$ and metaanalysis $[6,26-34]$ results supporting this finding.

In addition, a recent meta-analysis revealed that active smokers are at higher risk of mortality and serious complications [27]. Interestingly, this meta-analysis indicated that serious complications were observed in $48 \%$ of former smokers and $24 \%$ of current smokers during COVID-19 course [27]. This appears to be because former smokers have longer exposure times or accompanying diseases such as COPD due to smoking. This issue was explained recently in a rapid nonsystematic meta-analysis conducted by Guo FR, which showed that COPD increased the development of severe COVID-19 by 4.38 times [28]. These findings are supported by another rapid nonsystematic meta-analysis, suggesting that smoking increases the severity and mortality of COVID-19 in hospitalized patients [29]. In addition, a history of smoking has been reported to increase the progression of COVID-19 disease [30]. There is growing evidence to support WHO's statements that smokers are at a higher risk of developing severe COVID-19 and consequent death [31]. It seems very clear that the pandemic period is an opportunity to quit smoking due to the possibility of encountering worse clinical outcomes and complications in patients with smoking history [32].

The relationship between smoking and illness severity due to other respiratory viruses has been investigated 

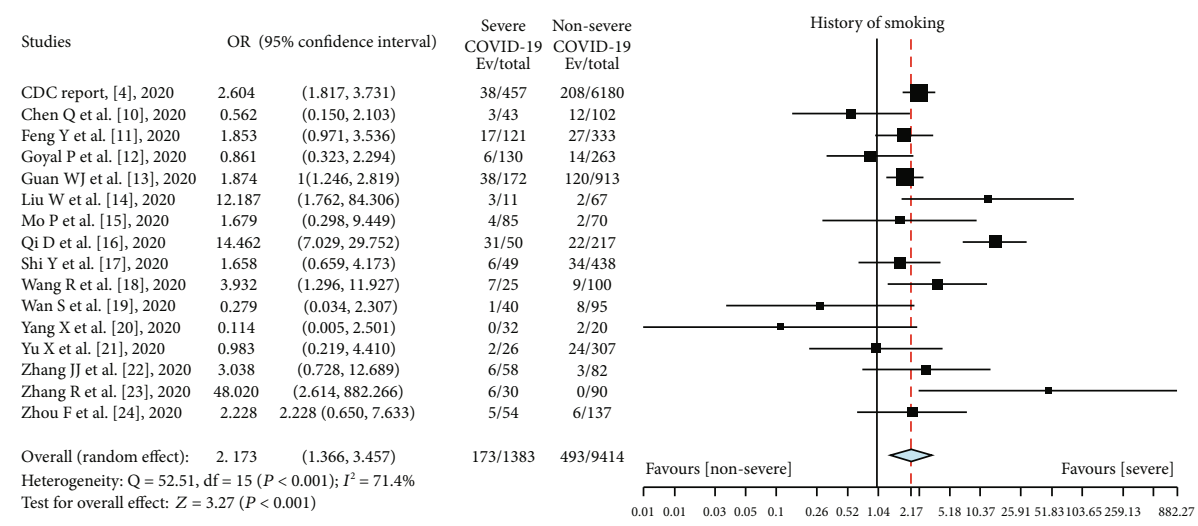

(a)

Funnel plot with pseudo $95 \%$ confidence limits

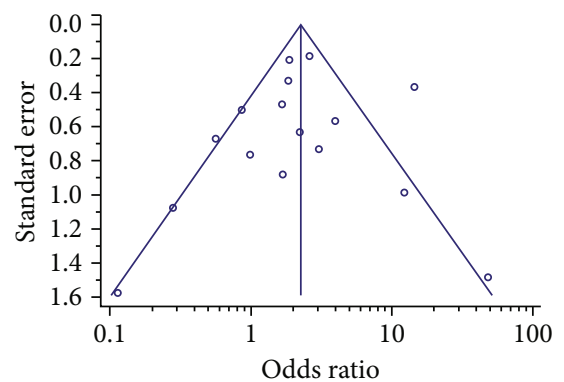

(b)

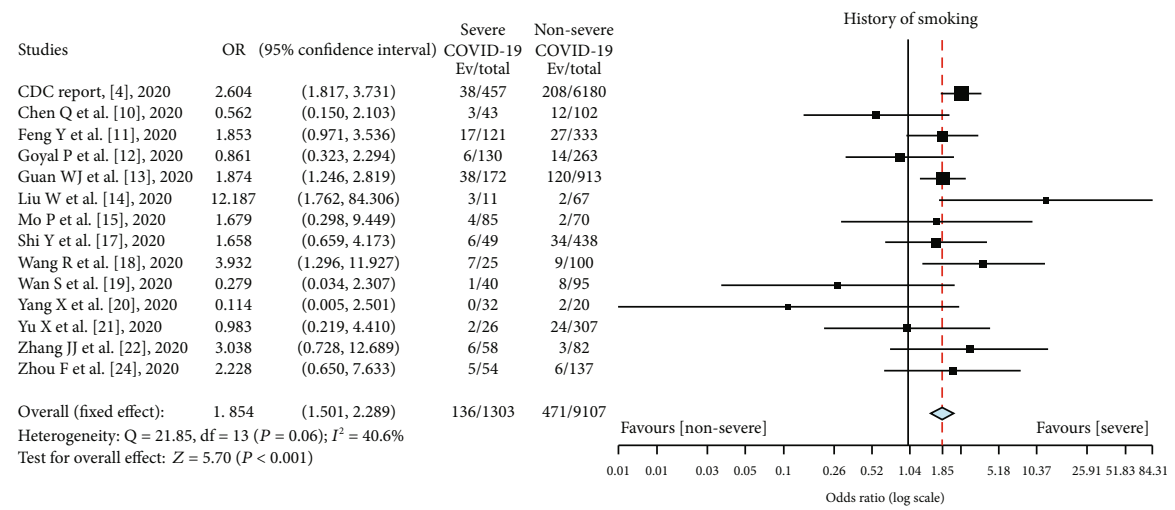

(c)

Funnel plot with pseudo 95\% confidence limits

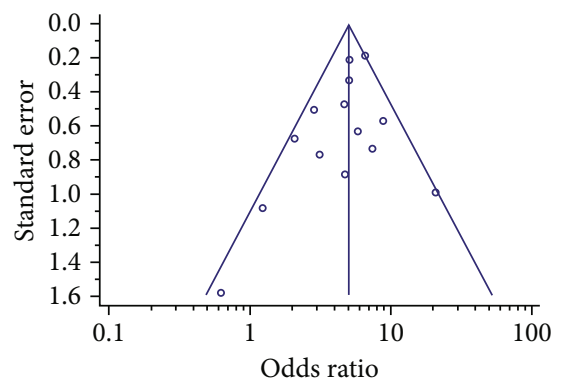

(d)

Figure 2: (a) Prevalence of smoking in severe COVID-19 patients. (b) Funnel plot for meta-analysis of the prevalence of smoking in severe COVID-19 patients. (c) Prevalence of smoking among severe COVID-19 patients after the exclusion of studies by Qi et al. [16] and Zhang et al. [23]. (d) Funnel plot for the meta-analysis of smoking among severe COVID-19 patients after the exclusion of studies by Qi D et al. [16] and Zhang R et al. [23]. 
TABLE 4: The relationship between smoking status and the severe COVID-19.

\begin{tabular}{|c|c|c|c|c|c|c|}
\hline \multirow{2}{*}{$\begin{array}{l}\text { Studies } \\
\text { Authors }\end{array}$} & \multicolumn{3}{|c|}{ Current smokers with severe COVID-19 } & \multicolumn{3}{|c|}{ Nonsmokers with severe COVID-19 } \\
\hline & Event & Total & $\%$ & Event & Total & $\%$ \\
\hline CDC report $[4]$ & 5 & 96 & 8.3 & 419 & 6901 & 60.7 \\
\hline Goyal et al. [12] & 6 & 20 & 30.0 & 124 & 373 & 33.2 \\
\hline Guan et al. [13] & 29 & 137 & 21.1 & 134 & 927 & 14.4 \\
\hline Mo et al. [15] & 4 & 6 & 66.6 & 81 & 149 & 54.3 \\
\hline Wang et al. [18] & 7 & 16 & 43.7 & 18 & 109 & 16.5 \\
\hline Wan et al. [19] & 1 & 9 & 11.1 & 39 & 126 & 30.9 \\
\hline Yang et al. [20] & 0 & 2 & 0 & 32 & 50 & 64.0 \\
\hline Zhang et al. [22] & 2 & 2 & 100 & 58 & 138 & 42.0 \\
\hline Zhang et al. [23] & 6 & 6 & 100 & 24 & 114 & 21.0 \\
\hline Zhou et al. [24] & 5 & 11 & 45.4 & 49 & 180 & 27.2 \\
\hline Total & 65 & 305 & 21.3 & 978 & 9067 & 10.7 \\
\hline
\end{tabular}

previously. In one recent study, COPD patients who smoked had decreased leukemia inhibitory factor levels during respiratory syncytial viral infection, particularly during exacerbations, which may have contributed to lung tissue injury [35]. In addition, smoking may reduce the protective effect of influenza vaccines in elderly patients and increase the risk of hospitalization [36]. As a result, adverse effects on the lungs in smokers may aggravate the symptom severity of viral infections.

Furthermore, novel SARS-CoV-2 uses an angiotensinconverting enzyme (ACE)-2 in the lungs to enter cells and cause infection. In patients using ACE inhibitors or angiotensin II receptor blockers (ARBs) for hypertension, upregulated ACE2 expression impacts on disease course remains unclear. However, the Council on Hypertension of the European Society of Cardiology does not recommend discontinuation of ACEI and ARB medications for all patients [37]. The ACE-2 upregulation may increase infectiousness and therefore infection risk, as the SARSCOV-2 virus uses this receptor for host entry. Paradoxically, it is stated that it may be useful in protecting people from acute lung injury [38].

In one recent study, ACE-2 gene expression was upregulated in the airway epithelium of COPD patients and active smokers, thereby suggesting a mechanism by which risk for severe COVID-19 increases in smokers [39]. This upregulation may occur through the $\alpha 7$ subtype of nicotine acetylcholine receptors $(\alpha 7-\mathrm{nAChR})$ in people who smoke and consequently consume nicotine [40]. Therefore, $\alpha 7-\mathrm{nAChR}$ antagonists (e.g., $\alpha$-conotoxin, methyllycaconitine), which may be used to assist with smoking cessation, should be investigated for their potential impact on ACE-2 expression and thus SARS-COV-2 entry into the host $[41,42]$. Furthermore, $\alpha 7-\mathrm{nAChR}$ and ACE- 2 may be potential therapeutic targets, a topic which requires further investigation.

Common comorbidities including cardiovascular disease, chronic kidney disease, diabetes, and hypertension are associated with increased risk for severe COVID-19 [43]. Similarly, COPD is also associated with a poor clinical progression and poor outcomes despite treatment, and it was reported that the risk of severe COVID-19 was observed 4 times higher in patients with preexisting COPD than in patients without COPD [6]. This may be explained by systemic and chronic inflammation, diminished respiratory function and capacity, and COPD-related respiratory failure in some patients. The prevalence of COPD was previously reported to be $2.8 \%$ in nonsmokers, $7.6 \%$ in former smokers, and $15.2 \%$ in active smokers [44]. Given this, smoking and COPD comorbidity should be considered together as a single risk factor for severe COVID-19.

Another important point for consideration is that acute respiratory distress syndrome and cytokine storms, which are seen in some severe COVID-19 patients, are key causes of mortality in COVID-19. In these patients, proinflammatory cytokines such as interleukin (IL)-1, IL-2, IL-6, IL-8, IL-17, interferon- $\gamma$, and TNF- $\alpha$ are elevated, which affect patients clinical symptoms and severity [45]. Nicotine is a cholinergic agonist that acts via the a7-nAChR pathway and also inhibits proinflammatory cytokines such as IL-1, IL-6, and TNF, but has no effect on IL-10, an antiinflammatory cytokine [46]. Therefore, nicotine may be beneficial for the treatment of severe COVID-19 when delivered via chewing gum, inhaler/aerosol, or patch $[47,48]$. This potential therapeutic application should be clarified with future clinical research, however.

Several factors limit the interpretation of the present study. First, the vast majority of studies included here were retrospective epidemiological studies conducted in China. Second, some included studies did not distinguish between former or current smoking status. Third, studies classified COVID-19 cases broadly as follows: (i) mild to moderate: mild, nonsevere, common type, did not require ICU care, and COVID-19 survivors and (ii) severe: severe, critical, required ICU care, and nonsurvivors. Given these limitations, caution should be exercised while interpreting our results. Future studies may respond to these issues by defining disease severity more clearly and by obtaining more detailed information about smoking habit.

In addition to classical tobacco smoking behavior, water pipe and electronic cigarette use should not be overlooked, as these modes of consumption may increase contamination risk due to repetitive hand interactions with the mouth, 

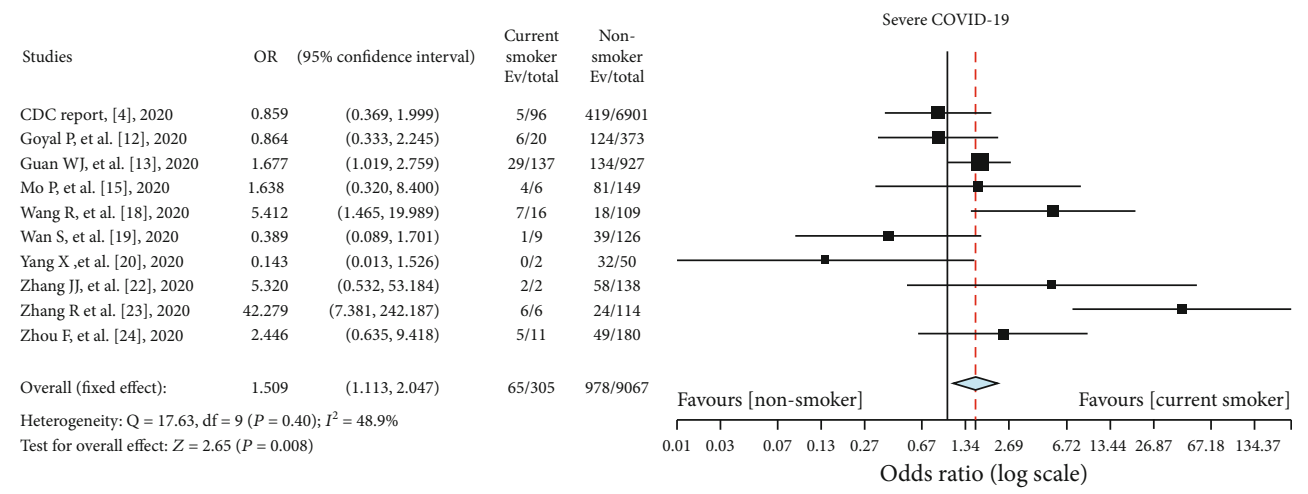

(a)

Funnel plot with pseudo 95\% confidence limits

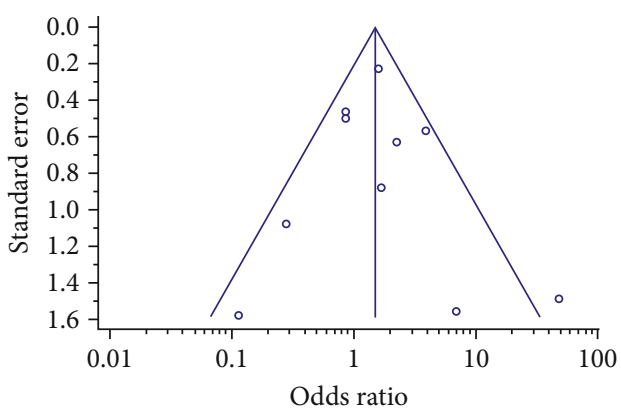

(b)

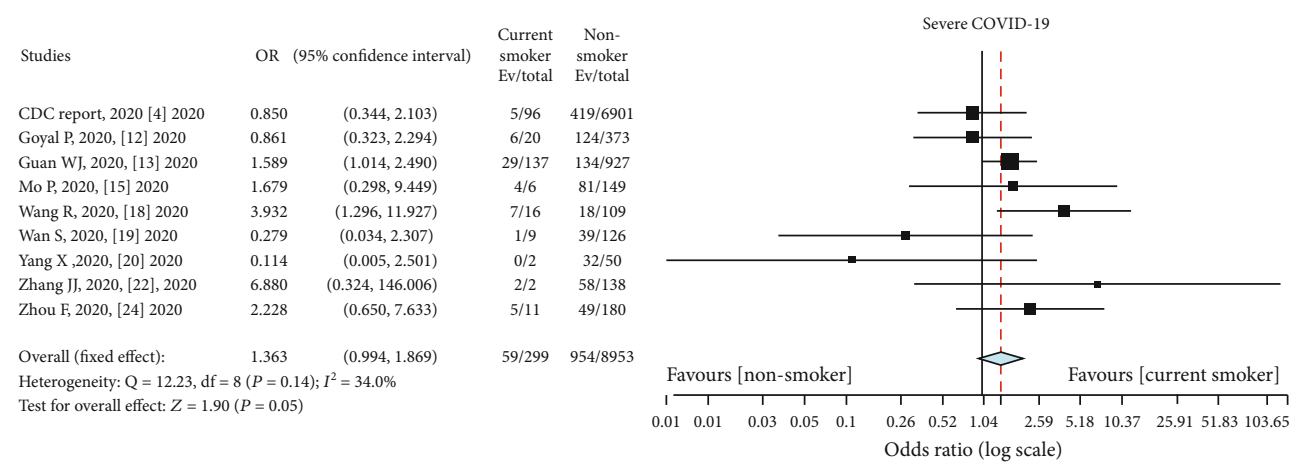

(c)

Funnel plot with pseudo 95\% confidence limits

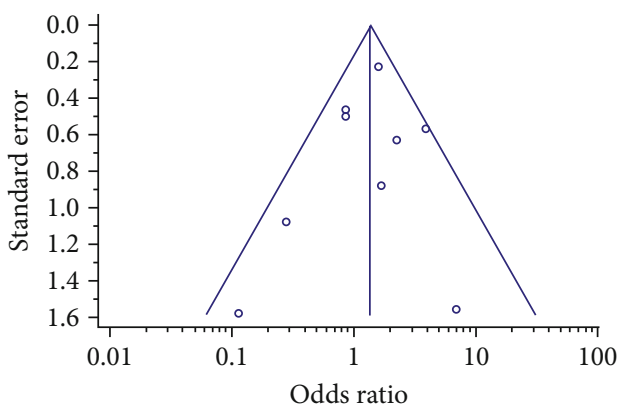

(d)

Figure 3: (a) Comparison of smoking status in severe COVID-19 patients. (b) Funnel plot for the meta-analysis of smoking among severe COVID-19 patients. (c) Comparison of smoking status among severe COVID-19 patients after the exclusion of a study by Zhang et al. [23]. (d) Funnel plot for the meta-analysis of smoking among severe COVID-19 patients after the exclusion of a study by Zhang et al. [23]. 
carrying cigarette packets in the pockets, and blowing of smoke. Similarly, exposure to passive smoke can alter ACE2 gene expression and cause immune system changes. Naturally, these patients also have the potential to have severe COVID-19 symptoms, and smoke exposure is a potential risk factor for those around the patient, including their friends and family.

Future studies should continue to collect nicotine consumption information, including the number of cigarettes smoked per day, passive exposure, and degree of COPD, and should evaluate the dynamics of interactions between cigarette smoking and COVID-19. In addition, the effects of various smoking habits (e.g., mild versus heavy consumption, water pipe use, and electronic cigarettes use) on the transmission of SARS-COV-2, the clinical severity of COVID-19, and the clinical progression of COVID-19 should be investigated. Finally, the relationship between COPD severity and COVID-19, and the potential therapeutic effect of nicotine on severe COVID patients should also be examined and clarified. In addition, clinicians can pay more attention to the history of smoking of COVID-19 patients, and more further research may aim to determine mechanisms that drive or decrease this risk.

\section{Conclusion}

The present meta-analysis revealed that active smoking and a history of smoking are significantly associated with increased COVID-19 symptom severity. The SARS-COV-2 epidemic should serve as an impetus for patients and those at risk to maintain good health practices and discontinue smoking.

\section{Data Availability}

The data [data.oma and data.xls] used to support the findings of this study are available from the corresponding author upon request.

\section{Conflicts of Interest}

The authors have stated explicitly that there are no conflicts of interest in connection with this article.

\section{Authors' Contributions}

AG performed the concept of the manuscript, data collection, design of tables and figures, analysis and interpretation of data, and writing and drafting of the manuscript; BU performed the data collection and quality analysis; BAY performed the acquisition of data, quality analysis, and writing and revising the manuscript; DD and OK performed the major role in reviewing and revising the manuscript.

\section{Acknowledgments}

Funding of the Federal Ministry for Science and Education (BMBF), German Center for Lung Research, is gratefully acknowledged.

\section{References}

[1] World Health Organisation, "Coronavirus disease (COVID2019) situation reports," https://www.who.int/docs/defaultsource/coronaviruse/situation-reports/20200824-weekly-epiupdate.pdf?sfvrsn=806986d1_4.

[2] Coronavirus Worldometer, "Country-specific case fatality rates (CFR) of COVID-19 patients," August 2020 https:// www.worldometers.info/coronavirus/.

[3] J. Yang, Y. Zheng, X. Gou et al., "Prevalence of comorbidities and its effects in patients infected with SARS-CoV-2: a systematic review and meta-analysis," International Journal of Infectious Diseases, vol. 94, pp. 91-95, 2020.

[4] CDC COVID-19 Response Team, CDC COVID-19 Response Team, N. Chow et al., "Preliminary Estimates of the Prevalence of Selected Underlying Health Conditions Among Patients with Coronavirus Disease 2019- United States, February 12March 28, 2020," MMWR. Morbidity and Mortality Weekly Report, vol. 69, no. 13, pp. 382-386, 2020.

[5] S. Garg, L. Kim, M. Whitaker et al., "Hospitalization Rates and Characteristics of Patients Hospitalized with LaboratoryConfirmed Coronavirus Disease 2019 - COVID-NET, 14 States, March 1-30, 2020," MMWR. Morbidity and Mortality Weekly Report, vol. 69, no. 15, pp. 458-464, 2020.

[6] Q. Zhao, M. Meng, R. Kumar et al., "The impact of COPD and smoking history on the severity of COVID-19: A systemic review and meta-analysis," Journal of Medical Virology, 2020.

[7] G. Lippi and B. M. Henry, "Active smoking is not associated with severity of coronavirus disease 2019 (COVID-19)," European Journal of Internal Medicine, vol. 75, pp. 107-108, 2020.

[8] D. Moher, A. Liberati, J. Tetzlaff, D. G. Altman, and for the PRISMA Group, "Preferred reporting items for systematic reviews and meta-analyses: the PRISMA statement," $B M J$, vol. 339, article b2535, 2009.

[9] G. A. Wells, B. Shea, D. O'Connell et al., The Newcastle-Ottawa Scale (NOS) for assessing the quality of nonrandomised studies in meta-analysesOttawa Hospital Research InstituteMay 2020 http://www.ohri.ca/programs/clinical_epidemiology/oxford.asp.

[10] Q. Chen, Z. Zheng, C. Zhang et al., "Clinical characteristics of 145 patients with corona virus disease 2019 (COVID-19) in Taizhou, Zhejiang, China," Infection, vol. 48, no. 4, pp. 543$551,2020$.

[11] Y. Feng, Y. Ling, T. Bai et al., "COVID-19 with Different Severities: A Multicenter Study of Clinical Features," American Journal of Respiratory and Critical Care Medicine, vol. 201, no. 11, pp. 1380-1388, 2020.

[12] P. Goyal, J. J. Choi, L. C. Pinheiro et al., "Clinical Characteristics of Covid-19 in New York City," The New England Journal of Medicine, vol. 382, no. 24, pp. 2372-2374, 2020.

[13] W. J. Guan, Z. Y. Ni, Y. Hu et al., "Clinical Characteristics of Coronavirus Disease 2019 in China," New England Journal of Medicine, vol. 382, no. 18, pp. 1708-1720, 2020.

[14] W. Liu, Z. W. Tao, L. Wang et al., "Analysis of factors associated with disease outcomes in hospitalized patients with 2019 novel coronavirus disease," Chinese Medical Journal, vol. 133, no. 9, pp. 1032-1038, 2020.

[15] P. Mo, Y. Xing, Y. Xiao et al., "Clinical characteristics of refractory COVID-19 pneumonia in Wuhan, China," Clinical Infectious Diseases, 2020.

[16] Q. Di, X. Yan, X. Tang et al., "Epidemiological and clinical features of 2019-nCoV acute respiratory disease cases in 
Chongqing municipality, China: a retrospective, descriptive, multiple-center study," medRxiv, 2020.

[17] Y. Shi, X. Yu, H. Zhao, H. Wang, R. Zhao, and J. Sheng, "Host susceptibility to severe COVID-19 and establishment of a host risk score: findings of 487 cases outside Wuhan," Critical Care, vol. 24 , no. $1,2020$.

[18] R. Wang, M. Pan, X. Zhang et al., "Epidemiological and clinical features of 125 Hospitalized Patients with COVID-19 in Fuyang, Anhui, China," International Journal of Infectious Diseases, vol. 95, pp. 421-428, 2020.

[19] S. Wan, Y. Xiang, W. Fang et al., "Clinical features and treatment of COVID-19 patients in northeast Chongqing," Journal of Medical Virology, vol. 92, no. 7, pp. 797-806, 2020.

[20] X. Yang, Y. Yu, J. Xu et al., "Clinical course and outcomes of critically ill patients with SARS-CoV-2 pneumonia in Wuhan, China: a single-centered, retrospective, observational study," The Lancet Respiratory Medicine, vol. 8, no. 5, pp. 475-481, 2020.

[21] X. Yu, X. Sun, P. Cui et al., "Epidemiological and clinical characteristics of 333 confirmed cases with coronavirus disease 2019 in Shanghai, China," Transboundary and Emerging Diseases, vol. 67, no. 4, pp. 1697-1707, 2020.

[22] J. J. Zhang, X. Dong, Y. Y. Cao et al., "Clinical characteristics of 140 patients infected with SARS-CoV-2 in Wuhan, China," Allergy, vol. 75, no. 7, pp. 1730-1741, 2020.

[23] R. Zhang, H. Ouyang, L. Fu et al., "CT features of SARS-CoV-2 pneumonia according to clinical presentation: a retrospective analysis of 120 consecutive patients from Wuhan city," European Radiology, vol. 30, no. 8, pp. 4417-4426, 2020.

[24] F. Zhou, T. Yu, R. Du et al., "Clinical course and risk factors for mortality of adult inpatients with COVID-19 in Wuhan, China: a retrospective cohort study," Lancet, vol. 395, no. 10229, pp. 1054-1062, 2020.

[25] K. Farsalinos, A. Barbouni, and R. Niaura, "Systematic review of the prevalence of current smoking among hospitalized COVID-19 patients in China: could nicotine be a therapeutic option?," Internal and Emergency Medicine, vol. 15, no. 5, pp. 845-852, 2020.

[26] A. Emami, F. Javanmardi, N. Pirbonyeh, and A. Akbari, "Prevalence of Underlying Diseases in Hospitalized Patients with COVID-19: a Systematic Review and Meta-Analysis," Archives of Academic Emergency Medicine, vol. 8, no. 1, article e35, 2020.

[27] J. S. Alqahtani, T. Oyelade, A. M. Aldhahir et al., "Prevalence, Severity and Mortality associated with COPD and Smoking in patients with COVID-19: A Rapid Systematic Review and Meta-Analysis," PLOS ONE, vol. 15, no. 5, article e0233147, 2020.

[28] F. R. Guo, "Smoking links to the severity of COVID-19: An update of a meta-analysis," Journal of Medical Virology, 2020.

[29] A. Karanasos, K. Aznaouridis, G. Latsios et al., "Impact of Smoking Status on Disease Severity and Mortality of Hospitalized Patients With COVID-19 Infection: A Systematic Review and Meta-analysis," Nicotine \& Tobacco Research, 2020.

[30] R. Patanavanich and S. A. Glantz, "Smoking is Associated with COVID-19 Progression: A Meta-Analysis," Nicotine \& Tobacco Research, 2020.

[31] E. Grundy, T. Suddek, F. T. Filippidis, A. Majeed, and S. Coronini-Cronberg, "Smoking, SARS-CoV-2 and COVID19: A review of reviews considering implications for public health policy and practice," Tobacco Induced Diseases, vol. 18, 2020.
[32] R. K. Reddy, W. N. Charles, A. Sklavounos, A. Dutt, P. T. Seed, and A. Khajuria, "The effect of smoking on COVID-19 severity: A systematic review and meta-analysis," Journal of Medical Virology, 2020.

[33] K. Farsalinos, A. Barbouni, K. Poulas, R. Polosa, P. Caponnetto, and R. Niaura, "Current smoking, former smoking, and adverse outcome among hospitalized COVID19 patients: a systematic review and meta-analysis," Therapeutic Advances in Chronic Disease, vol. 11, 2020.

[34] D. C. Sanchez-Ramirez and D. Mackey, "Underlying respiratory diseases, specifically COPD, and smoking are associated with severe COVID-19 outcomes: A systematic review and meta-analysis," Respiratory Medicine, vol. 171, article 106096, 2020.

[35] J. Poon, M. Campos, R. F. Foronjy et al., "Cigarette smoke exposure reduces leukemia inhibitory factor levels during respiratory syncytial viral infection," International Journal of Chronic Obstructive Pulmonary Disease, vol. 14, pp. 1305$1315,2019$.

[36] P. Godoy, J. Castilla, N. Soldevila et al., "Smoking may increase the risk of influenza hospitalization and reduce influenza vaccine effectiveness in the elderly," European Journal of Public Health, vol. 28, no. 1, pp. 150-155, 2018.

[37] European Society of Cardiology, "Position Statement of the ESC Council on Hypertension on ACE-Inhibitors and Angiotensin Receptor Blockers," March 2020 https://www.escardio .org/Councils/Council-on-Hypertension-(CHT)/News/ position-statement-of-the-esc-council-on-hypertension-onace-inhibitors-and-ang.

[38] S. H. Javanmard, K. Heshmat-Ghahdarijani, and G. Vaseghi, "Angiotensin-converting-enzyme inhibitors (ACE inhibitors) and angiotensin II receptor blocker (ARB) use in COVID-19 prevention or treatment: A paradox," Infection Control and Hospital Epidemiology, pp. 1-2, 2020.

[39] J. M. Leung, C. X. Yang, A. Tam et al., “ACE-2 expression in the small airway epithelia of smokers and COPD patients: implications for COVID-19," European Respiratory Journal, vol. 55, no. 5, article 2000688, 2020.

[40] P. Russo, S. Bonassi, R. Giacconi, M. Malavolta, C. Tomino, and F. Maggi, "COVID-19 and smoking: is nicotine the hidden link?," European Respiratory Journal, vol. 55, no. 6, article 2001116, 2020

[41] J. M. Leung, C. X. Yang, and D. D. Sin, "COVID-19 and nicotine as a mediator of ACE-2," European Respiratory Journal, vol. 55, no. 6, article 2001261, 2020.

[42] L. Ramachandran Nair and X. Liu, "Targeting the $\alpha 4 \beta 2$ - and $\alpha 7$-Subtypes of Nicotinic Acetylcholine Receptors for Smoking Cessation Medication Development," Journal of Addiction Research \& Therapy, vol. 10, no. 2, p. 381, 2019.

[43] X. Wang, X. Fang, Z. Cai et al., "Comorbid Chronic Diseases and Acute Organ Injuries Are Strongly Correlated with Disease Severity and Mortality among COVID-19 Patients: A Systemic Review and Meta-Analysis," Research, vol. 2020, pp. 1-17, 2020.

[44] A. G. Wheaton, Y. Liu, J. B. Croft et al., "Chronic Obstructive Pulmonary Disease and Smoking Status - United States, 2017," MMWR. Morbidity and Mortality Weekly Report, vol. 68, no. 24, pp. 533-538, 2019.

[45] D. McGonagle, K. Sharif, A. O'Regan, and C. Bridgewood, "The Role of Cytokines including Interleukin-6 in COVID-19 induced Pneumonia and Macrophage Activation Syndrome- 
Like Disease," Autoimmunity Reviews, vol. 19, no. 6, article 102537, 2020.

[46] L. Ulloa, "The vagus nerve and the nicotinic anti-inflammatory pathway," Nature Reviews. Drug Discovery, vol. 4, no. 8, pp. 673-684, 2005.

[47] S. Zi, J. Li, L. Liu, and F. Liu, "Cholinergic anti-inflammatory pathway and its role in treatment of sepsis," Journal of Central South University. Medical Sciences, vol. 45, no. 1, pp. 68-73, 2020.

[48] K. Farsalinos, R. Niaura, J. Le Houezec et al., "Editorial: Nicotine and SARS-CoV-2: COVID-19 may be a disease of the nicotinic cholinergic system," Toxicology Reports, vol. 7, pp. 658-663, 2020. 